\title{
Femtosecond laser 3D micromachining and its applications to biochip fabrication (Presentation Video)
}

\section{Koji Sugioka}

Koji Sugioka, "Femtosecond laser 3D micromachining and its applications to biochip fabrication (Presentation Video)," Proc. SPIE 8970, Laser 3D Manufacturing, 897007 (18 March 2014); doi: 10.1117/12.2063586 


\title{
Femtosecond Laser 3D Micromachining and its Applications to Biochip Fabrication (Presentation Video)
}

Koji Sugioka, RIKEN (Japan)

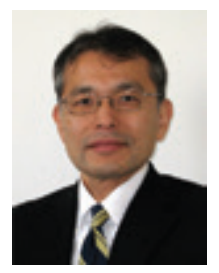

\begin{abstract}
Femtosecond lasers have opened up new avenues in materials processing due to their unique characteristics of ultra-short pulse widths and extremely high peak intensities that induce strong absorption in even transparent materials due to nonlinear multiphoton absorption. Then, the femtosecond laser can directly fabricate three-dimensional microfluidic, micromechanic, microelectronic, and micro-optical components in glass. These microcomponents can be easily integrated in a single glass microchip, which enable us to fabricate functional biochips quickly screening large number of biological analytes. In this talk, the detailed fabrication procedure of biochips using the femtosecond laser and applications of the fabricated biochips to material synthesis, analysis of biochemical samples, and determination of functions of microorganisms are introduced.
\end{abstract}

View presentation video on SPIE's Digital Library: http://dx.doi.org/10.1117/12.2063586 